\title{
Formación inicial de maestros: escenarios activos desde una perspectiva del aula invertida
}

\author{
Cesar A. Hernández-Suárez*, Raúl Prada-Núñez y Audin A. Gamboa-Suárez \\ Facultad de Educación, Artes y Humanidades, Universidad Francisco de Paula Santander, Cúcuta - Colombia \\ (correo-e: cesaraugusto@ufps.edu.co; raulprada@ufps.edu.co; audingamboa@ufps.edu.co) \\ ${ }^{*}$ Autor a quien debe ser dirigida la correspondencia
}

Recibido Feb. 17, 2020; Aceptado Abr. 20, 2020; Versión final May. 19, 2020; Publicado Oct. 2020

\begin{abstract}
Resumen
El presente artículo analiza el impacto en el rendimiento académico y las opiniones de los estudiantes en la implementación del modelo aula invertida en un programa de formación de docentes de matemáticas. El horizonte metodológico se enmarca en la investigación educativa empírica con el diseño teórico de los entornos de aprendizaje. Se aplicó una prueba pretest-postest y un cuestionario escala Likert. La muestra estuvo conformada por 42 estudiantes de un programa de pregrado de una universidad pública en Norte de Santander (Colombia). Los resultados evidencian que el rendimiento académico de los estudiantes en los que se utilizó esta metodología tiende a mejorar en comparación con los estudiantes que utilizan el método tradicional. Las opiniones de los estudiantes sobre esta estrategia fueron en su mayoría positivas, lo que demostró la preferencia por este escenario innovador. Este trabajo da un paso hacia la conceptualización del modelo aula invertida como un esfuerzo para transformar los cursos tradicionales.
\end{abstract}

Palabras clave: aula invertida; innovación pedagógica; aprendizaje activo; formación inicial de docentes

\section{Pre-service teacher education: active scenarios from a flipped classroom a perspective}

\begin{abstract}
This present study analyzes the impact of the flipped classroom model on student academic performance and opinions in a mathematics teacher-training program. The methodology was framed in educational - empirical research with the theoretical design of learning environments. A pre-test, a post-test, and a Likert scale questionnaire were applied. The sample consisted of 42 students from an undergraduate program of a public university in Norte de Santander (Colombia). The results show that academic performance of the students using the flipped classroom model improved when compared to students using a traditional method. In addition, students' opinions about the flipped classroom model were mostly positive, which showed a preference for this innovative scenario. The present study is a step forward towards conceptualizing the flipped classroom model as an initiative to transform traditional classes.
\end{abstract}

Keywords: flipped classroom; pedagogical innovation; active learning; pre-service teacher education 


\section{INTRODUCCIÓN}

El desafío para los docentes debe trascender más allá de la instrucción, su rol implica la construcción de aprendizaje. Pero, los avances en la tecnología, el crecimiento del contenido en línea disponible y los desarrollos en las ciencias cognitivas se combinan para desafiar los paradigmas tradicionales del proceso educativo (Gamboa, 2016). El modelo "aula invertida" (clase invertida, aprendizaje invertido) fue desarrollado para proporcionar los contenidos a los estudiantes que faltaban a clase (Bergmann y Sams, 2012).

El interés por el aula invertida ha proliferado en todos los niveles educativos en los últimos años; aunque tales prácticas se implementan con frecuencia con ligeras variaciones. En un escenario de aula invertida típica, lo que suele ser trabajo en clase (el profesor presenta los contenidos mientras los estudiantes toman notas) se realiza antes de esta; es decir, hay un ejercicio importante de trabajo autónomo, mientras que ahora estas actividades se implementan en clase y se usa este tiempo de manera efectiva para participar en actividades de aprendizaje activo, facilitadas por el docente en interacción con todos los estudiantes (Bergmann y Sams, 2012). Investigaciones han mostrado que los docentes han evidenciado las ventajas de invertir el aula, como una mayor participación y un mayor rendimiento de los estudiantes, así como cursos bien diseñados (O'Flaherty y Phillips, 2015; Zainuddin y Halili, 2016). La implementación del aula invertida en los diversos contextos educativos es de recién incorporación y de mayor interés para los investigadores

El aula invertida es un modelo pedagógico, que se compone de metodologías activas y que utiliza diversas técnicas de desarrollo del aprendizaje (Martín et al., 2017). Se fundamenta en 3 principios: (1) la perspectiva cognitiva de Piaget, especialmente con los principios del constructivismo fundamentado en la resolución de problemas, el aprendizaje activo y el aprendizaje colaborativo; (2) la perspectiva social de Vygotsky y su zona de desarrollo próximo de donde se deriva el aprendizaje cooperativo; (3) el aprendizaje experiencial de Kolb muy asociado a los estilos de aprendizaje. Lo anterior muestra por un lado la integración entre el proceso cognitivo y como se construye el conocimiento, reconociendo los aprendizajes previos de los estudiantes (Gaviria et al., 2019), y por otro, la relación que se establece entre aprendizaje colaborativo y cooperativo (Durley, 2014).

De esta manera, fuera del aula, los estudiantes trabajan por un lado de forma individual y a su ritmo, mientras que, en el aula, lo hacen de forma dinámica e interactiva, resuelve problemas, trabaja en grupo, los docentes no hacen explicaciones, si no que orientan, retroalimentan y los conducen a que se dé el aprendizaje a través de la interacción. Además, el estudiante debe disponer de una rubrica de evaluación; de esta manera, puede decidir, hasta dónde desea llegar (Bergmann y Sams, 2012). Lo anterior, obliga al docente a cambiar su rol, ya que la clase no gira en torno a la explicación y se hace efectiva cuando se adapta a los diferentes ritmos de aprendizaje y ningún estudiante se queda atrás, lo que mejora la relación profesor-estudiante. En el aula invertida, la responsabilidad del aprendizaje se transfiere del docente a los estudiantes a través de la participación en actividades interactivas y aprendizaje activo lo que permite mejorar su rendimiento académico (Cabı, 2018; Oliván et al., 2019; Collins, 2019).

El modelo de aprendizaje invertido proporciona un marco conciso pero inclusivo en su esfuerzo por contextualizar el diseño curricular, por ejemplo, en la enseñanza de las matemáticas. Hay diversos marcos conceptuales que presentan diferentes componentes de aprendizaje para que los docentes enmarquen su diseño curricular. Un ejemplo relevante es el propuesto por Prada et al. (2019a), sobre el aprendizaje invertido que integra el uso de plataformas virtuales para enriquecer el aprendizaje. Dado que actualmente se definen varios enfoques de manera amplia, es necesario que los docentes e investigadores diseñen marcos conceptuales específicos para abordar sus necesidades.

Los cuatro principios delineados en el modelo de aprendizaje invertido (Hamdan et al., 2013) llamados pilares FLIP (en español, Invertir), son: entornos flexibles (en inglés, Flexible environment), cultura de aprendizaje (en inglés, Learning culture), contenido intencional (en inglés, Intentional content) y educadores profesionales (en inglés, Professional educator). Una característica que resalta el aula invertida, en curso de matemáticas, es su capacidad para crear entornos de aprendizaje flexibles, primer pilar del modelo de aprendizaje invertido. Esto se evidencia cuando los aprendices estudian los materiales antes de la clase. Bajo esta condición, los estudiantes pueden decidir dónde, cuándo y cómo ven el material. La flexibilidad de acceder al material en cualquier lugar, en cualquier momento y a su propio ritmo es ideal para reducir los sentimientos de ansiedad y frustración que pueden impedir la adquisición de conceptos matemáticos (Verkijika y De Wet, 2015). Pero estos materiales deben ser comprensibles para los estudiantes. Por lo tanto, la instrucción debe garantizar un entorno flexible.

El segundo pilar establece que se transfiere la responsabilidad de los docentes a los estudiantes, lo que da como resultado un aprendizaje centrado en el estudiante. Esto implica un cambio en el rol docente al pasar de un transmisor del conocimiento a un orientador que comprende las necesidades de los estudiantes y facilita su participación en actividades de aprendizaje. El aprendizaje de las matemáticas, debe estar centrado en el desarrollo de competencias (Gravemeijer et al., 2017; Prada et al., 2020); por lo tanto, los docentes que 
adopten el modelo aula invertida, deben asegurarse de que la enseñanza se dirija a proporcionar a los estudiantes oportunidades interactivas para el máximo uso del tiempo de clase.

Otra consideración importante en el aula invertida es el uso de contenido intencional para fomentar el aprendizaje de los estudiantes en las actividades previas y durante la clase. Con el predominio del enfoque por competencias, se acepta que la instrucción debe proporcionar oportunidades para que los estudiantes se centren en el desarrollo de los procesos matemáticos y el contexto. Desde una perspectiva interaccionista los estudiantes deben estar respaldados por un diseño curricular cuidadoso y el desarrollo del contenido asociado al aula invertida de matemáticas.

El último pilar se refiere a la importancia del papel del profesor. Aunque una cantidad de enseñanza directa a menudo se reemplaza con materiales, los docentes en el aula invertida aún asumen un papel crucial porque toman decisiones curriculares sobre cuándo y cómo cambiar o responder a condiciones específicas para conocer mejor necesidades del estudiante. El surgimiento del modelo aula invertida para cursos de matemáticas y otras áreas encaja en lo planteado por autores como: Rivero-Guerra (2018); de Araujo et al. (2017), entre otros, para mejorar los cursos que se han enseñado de manera tradicional. Al hacerlo, los docentes pueden reconstruir sus formas de pensar y enseñar en respuesta a las demandas cambiantes de la profesión docente (Hernández et al., 2019).

Sin embargo, a pesar de los reconocidos beneficios, persisten ciertas preocupaciones y dudas con respecto al aula invertida (Cabı, 2018). Aunque el modelo parece completo y plausible, y si bien existe la tecnología para implementar el aula invertida, su relevancia para el contexto de la enseñanza de las matemáticas no se ha establecido a profundidad; por ejemplo, en el contexto latino hay pocos antecedentes empíricos sobre el uso o la eficacia de esta estrategia para mejorar el rendimiento de los estudiantes en matemáticas (Turra et al., 2019), mientras que en otros contextos parece haber recibido más atención para la educación en ciencia, tecnología, ingeniería y matemáticas (Hung, 2017). El trabajo de Kwan y colaboradores se destaca porque proporcionaron un diseño general y una justificación bien articulada del modelo aula invertida en la enseñanza de las matemáticas (Kwan et al., 2017). Por lo tanto, es necesario dilucidar cómo se puede aplicar el modelo aula invertida a la educación matemática antes de que los educadores puedan adoptar definitivamente las aulas invertidas.

De particular interés para este estudio, fue la viabilidad de utilizar el modelo aula invertida para rediseñar y transformar un curso de matemáticas de un programa de formación docente a nivel universitario, con la intención de actualizar la formación inicial de educadores y ofrecer modelos innovadores que modernicen las practicas pedagógicas (necesidades de formación experiencial), para adaptarlas a los futuros estudiantes, donde mejoren la relación estudiante-profesor, motiven hacia el aprendizaje de las matemáticas y valoren las potencialidades, fortalezas y debilidades de su uso, en búsqueda de una educación adaptada a los requerimientos actuales (Carmona-Mesa y Villa-Ochoa, 2017; Mendoza et al., 2019). Finalmente, con esto se busca establecer una relación dialógica entre lo pedagógico, disciplinar y tecnológico (Arévalo et al., 2019; Mendoza et al., 2019).

En consecuencia, este artículo informa sobre el potencial pedagógico del aula invertida que implicó el desarrollo de unos principios de diseño, para guiar la práctica pedagógica de un curso de matemáticas de un programa de formación docente. La hipótesis que se planteó sugirió que el modelo aula invertida tendría un efecto positivo en el rendimiento académico y las opiniones de los estudiantes. En consecuencia, su objetivo fue determinar el efecto del modelo aula invertida en el rendimiento académico y las opiniones de los estudiantes de un programa de formación de docentes. Para determinar sus opiniones se aplica un cuestionario tipo Likert que busca responder al interrogante ¿cómo determinar la influencia de los factores pedagógicos y de diseño del aula invertida acerca de la satisfacción, el aprendizaje, y la disposición?

\section{MÉTODO}

Se seleccionó la investigación basada en el diseño (IBD) o design based research (DBR) como guía metodológica, la cual tiene su origen en los trabajos de Collins (1992) y Brown (1992); y se fundamentó como metodología por Barab (2006). En el ámbito iberoamericano es difundido por Rinaudo y Donolo (2010). La denominación investigación basada en el diseño, sugiere que predomina la investigación y que se fundamenta en un proceso de diseño (Bakker y van Eerde, 2015). La IBD, se define como una metodología que combina la investigación educativa empírica con el diseño teórico de los entornos de aprendizaje, para comprender cómo, cuándo y por qué las innovaciones educativas funcionan en la práctica (Design-Based Research Collective, 2003). Es decir, tiene un doble objetivo; por un lado, se considera apropiado para mejorar la implementación del modelo aula invertida y por el otro lado avanzar en el marco conceptual del modelo de aprendizaje invertido adoptado en el presente estudio. 
En la IBD, se distinguen tres fases: (1) la preparación del experimento, (2) el experimento de enseñanza y (3) el análisis retrospectivo. En la fase 2 se efectúan las intervenciones en el aula y las sucesivas iteraciones de ciclos de tres pasos: diseño y formulación de hipótesis, intervención en el aula junto con la recogida de datos y análisis de los datos con el propósito de revisar y reformular la hipótesis.

\section{Población y muestra}

El sitio de investigación fue la Universidad Francisco de Paula Santander (Cúcuta - Colombia). La muestra estuvo representada por 42 estudiantes matriculados en la asignatura "TIC y Educación Matemática" del tercer semestre del programa de formación de maestros denominado "Licenciatura en Matemáticas", distribuidos de la forma siguiente: 21 estudiantes para el primer y segundo semestre académico, con una duración de 16 semanas cada uno, en el año 2018. Uno de los investigadores fue el docente del curso en los dos semestres.

\section{Diseño curricular}

La contrastación se desarrolló en la asignatura "TIC y Educación Matemática", con una intensidad de 2 horas por semana, más una (1) hora de trabajo independiente, para un total de 48 horas al semestre. Los requisitos del curso, el contenido y el docente se mantuvieron constantes para generar condiciones comparables. Antes del rediseño del curso, los estudiantes habían experimentado un aprendizaje combinado, clase presencial y virtual con la plataforma PLAD-UFPS (Prada et al., 2019b), pero ninguno de ellos había estudiado en aulas invertidas. La aplicación de la metodología IBD consistió en dos fases de acción, la primera funcionando como un curso normal (primer semestre de 2018), y la otra, el rediseño del curso con el modelo aula invertida (segundo semestre de 2018), como se puede ver en la Tabla 1.

\section{Instrumentos}

La recopilación de datos en la IBD permite triangular los resultados. El procedimiento de recopilación se describe a continuación: el curso normal se orientó de la misma forma con un examen final en la última semana de clase mientras que, al curso con aula invertida, se le administró una prueba diagnóstica la primera semana y un examen final la última semana de clase sobre el contenido desarrollado para evaluar el aprendizaje de los estudiantes como parte de los requisitos del curso, que sirvió como pretest - postest para hacer la comparación. Otra medida del progreso de los estudiantes del curso con aula invertida fue la comparación con los resultados del examen final del curso normal, que fue el mismo para ambos cursos. El examen estaba compuesto de tres secciones: preguntas de conocimiento $(20 \%)$, resolución de ejercicios con procedimiento $(30 \%)$ y resolución de situaciones problémicas $(50 \%)$. El puntaje total de cada estudiante se usó como una medida del rendimiento académico en el curso.

También se diseñó y aplicó un cuestionario tipo Likert para conocer la opinión de los estudiantes del curso con aula invertida acerca de su satisfacción, aprendizaje, y disposición con este modelo. El cuestionario contó con tres escalas (satisfacción, aprendizaje, y disposición hacia el aula invertida) y 5 opciones de respuesta, desde totalmente en desacuerdo (1) pasando por ni acepta ni rechaza (3) hasta totalmente de acuerdo (5), el cual se sometió al análisis de tres expertos (en TIC, Educación e Investigación respectivamente), para que emitieran juicios de validez de contenido de cada uno de los ítems incluidos; todos coinciden en afirmar que el cuestionario está bien diseñado porque las escalas, están relacionadas teóricamente con las dimensiones establecidas en el estudio. Para la recolección de la información se contó con el consentimiento informado de los estudiantes y se tuvo en cuenta la confidencialidad y anonimato de ellos. La encuesta se administró en línea utilizando Google Form, durante una semana, antes del examen final. La tasa de respuesta de la encuesta fue del 90\% (De un total de 21 estudiantes, 19 completaron voluntariamente el cuestionario).

\section{RESULTADOS}

Para medir la fiabilidad del instrumento, se utilizó el coeficiente alfa de Cronbach (a). Se trabajó con estudiantes con las mismas características similares al grupo objeto de estudio. De 18 estudiantes, se obtuvo una taza de respuesta del $82 \%$ para llegar a las medidas de confiabilidad: satisfacción $=0.82$; aprendizaje $=$ 0.82 y disposición $=0.83$, con valores superiores a 0,70 que indica un nivel de fiabilidad aceptable en la investigación. Para la comparación entre la prueba diagnóstica y el examen final en el curso con aula invertida, las estadísticas descriptivas para las evaluaciones fueron en el pretest $(1.7 \pm 0.6$, rango $0.0-5.0)$ y en el postest $(3.9 \pm 0.6$, rango $0.0-5.0)$. Una prueba $t$ pareada arrojó diferencias significativas para el rendimiento de los estudiantes entre pretest y postest, $p<0.001$. De este modo, se puede concluir que los resultados más positivos fueron los del postest. 
Tabla 1: Aplicación del diseño curricular

\begin{tabular}{|c|c|c|c|c|c|c|}
\hline \multicolumn{7}{|c|}{ Asignatura: TIC y educación matemática } \\
\hline Contenido: & \multicolumn{3}{|c|}{$\begin{array}{l}\text { - Función lineal y afín (Algebra). } \\
\text { - Semejanza: teorema de Thales } \\
\text { (Geometría). } \\
\text { - Medidas de tendencia central (Estadística). }\end{array}$} & \multicolumn{2}{|c|}{$\begin{array}{l}\text { Intensidad horaria semanal } \\
\text { (h) }\end{array}$} & $\begin{array}{l}\text { - } 2 \mathrm{~h} \text { de clase } \\
\text { - } 1 \mathrm{~h} \text { de asesoría }\end{array}$ \\
\hline $\begin{array}{l}\text { Distribución del } \\
\text { semestre } \\
\text { académico (en } \\
\text { semanas): }\end{array}$ & \multicolumn{3}{|c|}{$\begin{array}{l}\text { - Dos (2) para la introducción de la asignatura } \\
\text { - } 10 \text { para el desarrollo del contenido temático } \\
\text { - Dos (2) para evaluaciones intermedias } \\
\text { - Una (1) para el examen final }\end{array}$} & \multicolumn{2}{|l|}{ Recursos: } & $\begin{array}{l}\text { Material impreso } \\
\text { y digital }\end{array}$ \\
\hline \multicolumn{2}{|c|}{ Curso con aula normal } & \multicolumn{5}{|c|}{ Curso con aula invertida (II semestre de 2018) } \\
\hline \multicolumn{2}{|c|}{$\begin{array}{c}21 \text { estudiantes } \\
\text { matriculados en el I } \\
\text { semestre de } 2018\end{array}$} & \multicolumn{5}{|c|}{21 estudiantes matriculados en el I semestre de 2018} \\
\hline \multicolumn{2}{|c|}{$\begin{array}{l}\text { Descripción de una clase, } \\
\text { fundamentada en el } \\
\text { modelo pedagógico } \\
\text { institucional: }\end{array}$} & \multicolumn{5}{|c|}{ Descripción de la clase con el enfoque aula invertida: } \\
\hline \multirow{2}{*}{\multicolumn{2}{|c|}{ 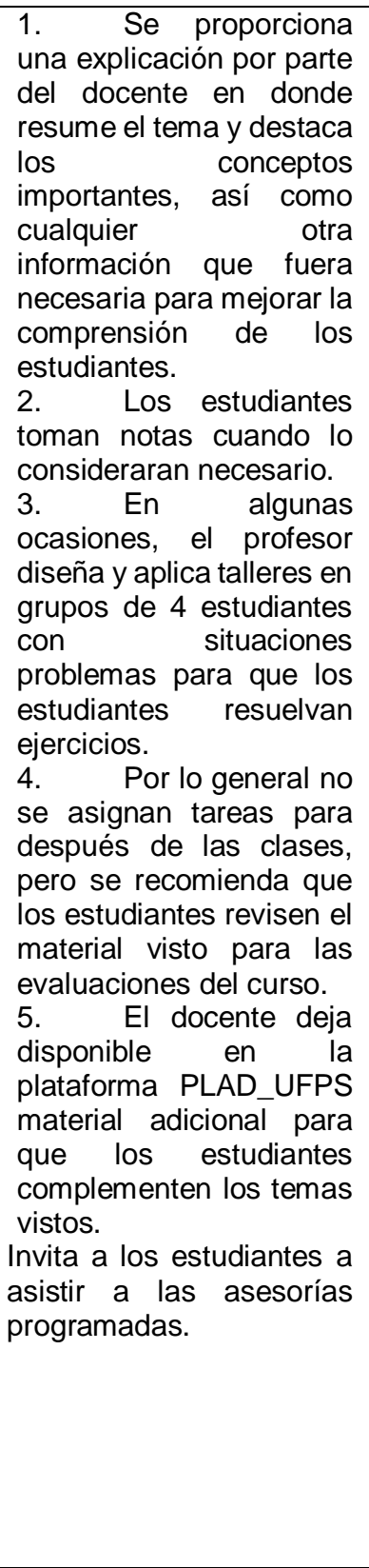 }} & Antes de la clase: & \multicolumn{2}{|r|}{ En la clase } & \multicolumn{2}{|c|}{ Después de la clase } \\
\hline & & 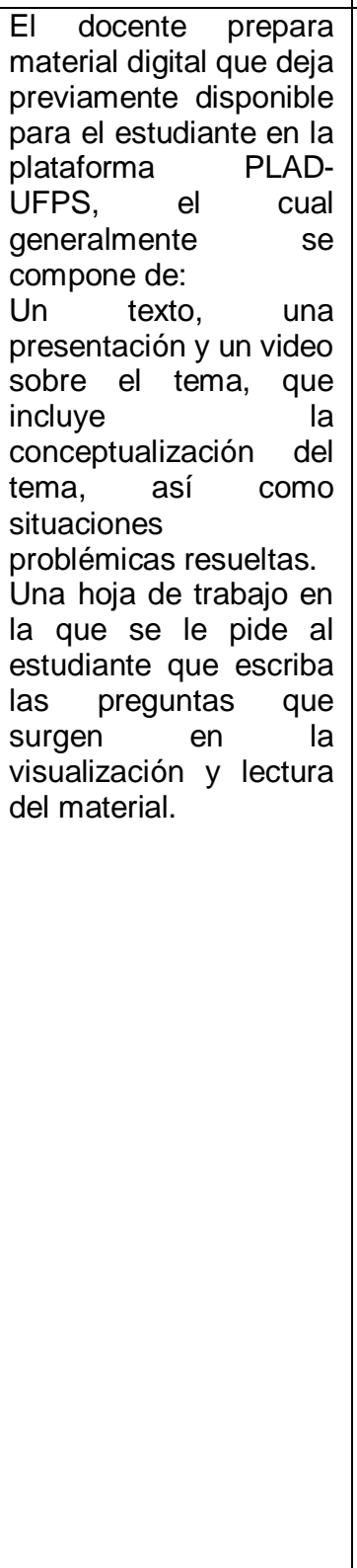 & \multicolumn{2}{|c|}{ 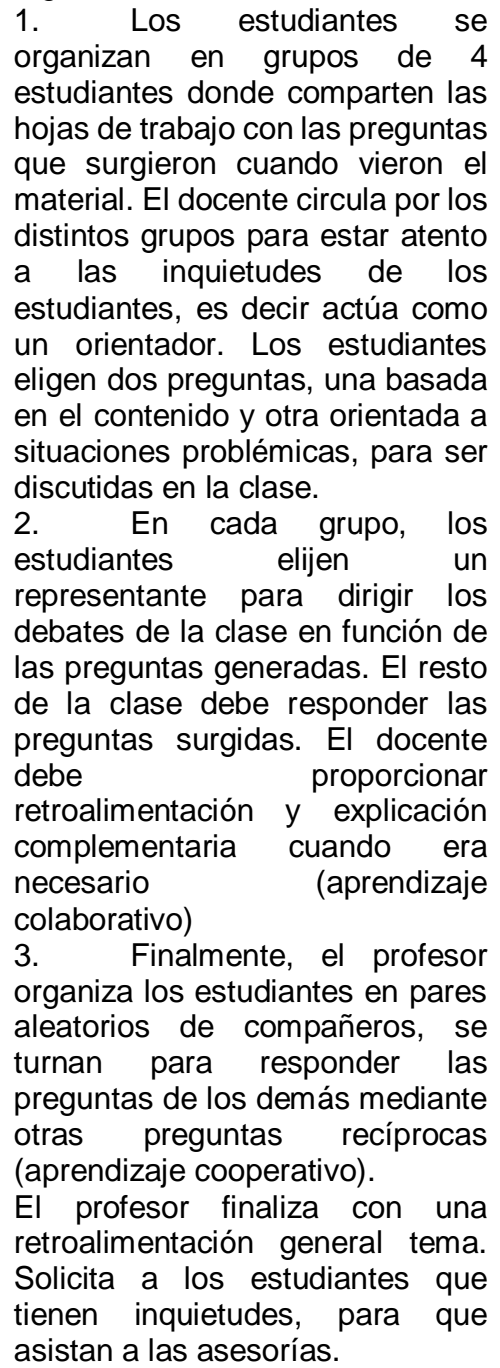 } & \multicolumn{2}{|c|}{$\begin{array}{l}\text { Los estudiantes } \\
\text { profundizan su } \\
\text { aprendizaje con } \\
\text { información adicional y } \\
\text { práctica de más } \\
\text { ejercicios. }\end{array}$} \\
\hline
\end{tabular}


Para determinar las diferencias en el rendimiento académico entre el curso normal y con aula invertida, se calcularon estadísticas descriptivas en el examen final de cada grupo, y se analizaron utilizando una prueba $t$ de muestras independientes. Se realizó una comparación entre grupos con el examen final. Las puntuaciones sumadas se calcularon y arrojaron estadísticas descriptivas para el curso normal (3.6 \pm 0.61 , [media $\pm D E$ ] rango $0.0-5.0$ ) y para el aula invertida (3.9 \pm 0.6 , rango $0.0-5.0)$. Una prueba $t$ que asumió la misma varianza para analizar las diferencias en el rendimiento de los estudiantes encontró diferencias significativas entre los exámenes finales del curso normal y de aula invertida, $p=0.024$. En general, los resultados sugieren que es más probable un mejor rendimiento en el curso con aula invertida.

Para la presentar las opiniones de los estudiantes, en la Tabla 2, se organizó la escala de respuesta con una reducción a tres niveles así: "de acuerdo" (totalmente de acuerdo y de acuerdo) y "en desacuerdo" (totalmente en desacuerdo y desacuerdo).

Tabla 2: Opiniones de los estudiantes del curso con aula invertida $(\mathrm{N}=19)$.

\begin{tabular}{|c|c|c|c|}
\hline Ítems & $\begin{array}{l}\text { Totalmente en } \\
\text { desacuerdo/ } \\
\text { En desacuerdo }\end{array}$ & Neutral & $\begin{array}{l}\text { De acuerdo/ } \\
\text { totalmente de } \\
\text { acuerdo }\end{array}$ \\
\hline \multicolumn{4}{|c|}{ Satisfacción (Media =4.31 / Desviación estándar = 0.37) } \\
\hline $\begin{array}{l}\text { El aula invertida es un espacio atractivo que facilita mí } \\
\text { aprendizaje. }\end{array}$ & $16(84.2 \%)$ & $2(10.6 \%)$ & $1(5.3 \%)$ \\
\hline El material digital se hizo comprensible para mí. & $14(73.5 \%)$ & $2(10.6 \%)$ & $3(15.9 \%)$ \\
\hline El profesor tenía conocimientos del modelo aula invertida. & $17(89.4 \%)$ & $2(10.6 \%)$ & $0(0.0 \%)$ \\
\hline Las actividades me ayudaron a lograr los objetivos del curso. & $13(68,4 \%)$ & $3(15,8 \%)$ & $3(15,8 \%)$ \\
\hline Estoy satisfecho y motivado para realizar las actividades. & $16(84.2 \%)$ & $2(10.6 \%)$ & $1(5.3 \%)$ \\
\hline Promedio \% & $80,0 \%$ & $11,6 \%$ & $8,4 \%$ \\
\hline \multicolumn{4}{|c|}{ Aprendizaje $($ Media $=4.12$ / Desviación estándar $=0.36)$} \\
\hline $\begin{array}{l}\text { El docente hizo conexiones significativas entre los materiales } \\
\text { digitales y la actividad de la clase. }\end{array}$ & $17(89.4 \%)$ & $1(5.3 \%)$ & $1(5.3 \%)$ \\
\hline $\begin{array}{l}\text { El docente requirió la participación del estudiante en las } \\
\text { actividades. }\end{array}$ & $15(78.8 \%)$ & $2(10.6 \%)$ & $2(10.6 \%)$ \\
\hline $\begin{array}{l}\text { El aula invertida me ayudo a desarrollar habilidades y resolver } \\
\text { problemas. }\end{array}$ & $12(63.2)$ & $3(15.8)$ & $4(21.1 \%)$ \\
\hline $\begin{array}{l}\text { En el aula invertida es fácil resolver las dudas y me permite } \\
\text { participar en clase. }\end{array}$ & $14(73.5 \%)$ & $2(10.6 \%)$ & $3(15.9 \%)$ \\
\hline $\begin{array}{l}\text { Tengo confianza en mi capacidad para abordar los temas en el } \\
\text { examen. }\end{array}$ & $15(78.8 \%)$ & $2(10.6 \%)$ & $2(10.6 \%)$ \\
\hline Promedio \% & $76.8 \%$ & $11.6 \%$ & $11.6 \%$ \\
\hline \multicolumn{4}{|c|}{ Disposición (Media =4.17 /Desviación estándar = 0.26) } \\
\hline $\begin{array}{l}\text { Ver el contenido y materiales digitales previamente fue } \\
\text { esencial y me preparó para la actividad de la clase. }\end{array}$ & $15(78.8 \%)$ & $2(10.6 \%)$ & $2(10.6 \%)$ \\
\hline $\begin{array}{l}\text { EL aula invertida favorece la interacción entre el profesor y los } \\
\text { estudiantes en comparación con aula normal. }\end{array}$ & $16(84.2 \%)$ & $2(10.6 \%)$ & $1(5.3 \%)$ \\
\hline El aula invertida es diferente a otras estrategias de clase. & $15(78.8 \%)$ & $3(15.9 \%)$ & $1(5.3 \%)$ \\
\hline Me gustaría que más docentes usaran el modelo aula invertida. & $12(63.2 \%)$ & $4(21.1 \%)$ & $3(15.8 \%)$ \\
\hline $\begin{array}{l}\text { Aprendí más cuando se utilizó el aula invertida en comparación } \\
\text { con el método tradicional. }\end{array}$ & $14(73.5 \%)$ & $3(15.9 \%)$ & $2(10.6 \%)$ \\
\hline Promedio \% & $75.8 \%$ & $14.7 \%$ & $9.5 \%$ \\
\hline
\end{tabular}

Con respecto a la escala de satisfacción del curso, los resultados, en forma global, revelaron que el grupo estaba muy satisfecho con el aula invertida $(80 \%)$. Por otra parte, las opiniones sobre el aprendizaje fueron favorables en el $76.8 \%$ de los estudiantes. Se destaca que en opinión del $78.8 \%$ de los estudiantes, consideran que el docente requiere de la participación de ellos; y que el $89.4 \%$ de los estudiantes estuvo de acuerdo en que el docente hizo conexiones significativas entre el material digital y las actividades de la clase.

Finalmente, también la disposición de los estudiantes fue favorable (75.8\%). Se menciona que: el $84.2 \%$ estuvo de acuerdo en que era deseable una mayor interacción entre profesores y estudiantes. Además, el $78.8 \%$ de los estudiantes estuvo de acuerdo con los siguientes aspectos: el aula invertida mejoró su autoeficacia para abordar los temas del examen, es diferente a otras estrategias de clase y ver los contenidos y materiales digitales previamente es esencial para las actividades en clase. Por último, el $63.2 \%$ expresó el deseo de que más docentes usen el modelo aula invertida. 


\section{DISCUSIÓN}

El estudio actual examinó los efectos de cambiar el aula normal por un aula invertida en curso de matemáticas de un programa de formación de docentes. Los resultados generales confirmaron la viabilidad y el potencial de esta estrategia con respecto a mejorar el rendimiento académico. Estos hallazgos confirman, que los efectos de la inversión del aula mejoran el aprendizaje de los estudiantes (Kwan et al., 2017; Turra et al., 2019). Además, los resultados sugieren que el enfoque de aula invertida tiene un efecto positivo en las opiniones de los estudiantes (Rivero-Guerra, 2018).

El diseño curricular de esta aula invertida incluye los principios FLIP (Hamdan et al., 2013), y el aprendizaje colaborativo-cooperativo (Durley, 2014) lo que promueve el desarrollo de habilidades y competencias (Gravemeijer et al., 2017). El estudiante actúa como responsable de su aprendizaje (Martín et al., 2017), gracias a las preguntas que surgen con la revisión del material digital, y que resuelven con los grupos en las discusiones de la clase; mientras el docente organiza actividades del aula, adaptándola a los diferentes ritmos de los estudiantes.

Para el docente en formación, el uso del invertida es satisfactoria, porque de acuerdo con los principios FLIP (Hamdan et al., 2013), crea un entorno flexible (F) de aprendizaje (L) que permite ver previamente los contenidos intencionales (I), para que en clase se consolide su participación e interacción con el docente y sus compañeros. En la transformación de este proceso participa no solo como estudiantes sino como profesional $(\mathrm{P})$ de la educación en formación que busca mejorar su práctica pedagógica.

Este estudio se suma a la creciente tendencia de los docentes de invertir las aulas en curso de matemáticas O'Flaherty y Phillips, 2015). Por lo tanto, es razonable suponer que se publicarán más resultados de investigaciones en el futuro inmediato sobre las condiciones de entornos de aprendizaje invertido.

Se pueden destacar algunas ventajas y desventajas del modelo aula invertida en la Tabla 3 y que complementan la propuesta de Prada et al. (2019a).

Tabla 3: Aspectos para destacar del curso con aula invertida.

\begin{tabular}{|l|l|}
\hline \multicolumn{1}{|c|}{ Ventajas } & \multicolumn{1}{|c|}{ Desventajas } \\
\hline $\begin{array}{l}\text { Aumenta y facilita la interacción y la comunicación entre } \\
\text { docentes y estudiantes }\end{array}$ & $\begin{array}{l}\text { Requiere dedicación, tiempo y compromiso del docente y } \\
\text { estudiante }\end{array}$ \\
\hline $\begin{array}{l}\text { Promueve el aprendizaje autónomo, la resolución de } \\
\text { problemas y el rol activo y protagónico de los estudiantes }\end{array}$ & $\begin{array}{l}\text { Si el estudiante no revisa el material digital previamente } \\
\text { obstaculiza el desarrollo del aula invertida }\end{array}$ \\
\hline $\begin{array}{l}\text { Reduce los tiempos en la entrega de trabajos de los } \\
\text { estudiantes y la retroalimentación del docente. }\end{array}$ & $\begin{array}{l}\text { El docente debe ser competente digitalmente para la } \\
\text { elaboración del material }\end{array}$ \\
\hline Favorece el aprendizaje cooperativo y colaborativo & Está sujeto a la calidad y disponibilidad del material digital \\
\hline Se adecua los estilos de aprendizaje de los estudiantes & Depende de la motivación y actitud del estudiante \\
\hline Permite profundidad en los temas & La estrategia sólo funciona con algunos contenidos \\
\hline Facilita el seguimiento del docente a los estudiantes & No se facilita para todos los estudiantes \\
\hline Mejora el ambiente de aula & No debe enfocarse en el docente ni la en la tecnología \\
\hline Prepara mejor al estudiante para la evaluación & No deben equivocarse los roles \\
\hline Permite reutilizar, revisar, y aumentar el material digital & \\
\hline
\end{tabular}

Con respecto a la metodología, Molina et al. (2011); O'Flaherty y Phillips, (2015) y Zainuddin y Halili (2016), sugieren realizar investigaciones basadas en el aula que permiten desarrollar comprensiones teóricas relevantes sobre enfoques prácticos para el desarrollo de pedagogías más efectivas, en este caso la implementación del aula invertida en un curso de matemática. La metodología adoptada hace eco de esta afirmación, con su doble objetivo de refinar tanto la teoría como la práctica de la pedagogía del enfoque de aula invertida (Design-Based Research Collective, 2003). Por lo tanto, se recomienda a los docentes que deseen mejorar sus prácticas pedagógicas de manera sistemática realicen la IBD, a fin de crecer profesionalmente como docentes e investigadores. Además, el IBD permitió el tránsito de un modelo transmisivo a un modelo centrado en las actividades de los estudiantes, lo que pone de relieve la necesidad de investigar aspectos relacionados con el diseño de entornos de aprendizaje y las prestaciones tecnológicas para incrementarlo.

Los hallazgos sugieren la posibilidad de seguir usando el modelo aula invertida en la formación inicial docente. Por un lado, como estudiantes, reflexionan sobre el compromiso con su propio aprendizaje y la necesidad de desarrollarlo de manera grupal y autónoma. Y por el otro, en calidad de docente en formación, analizan la importancia de modernizar las prácticas pedagógicas con modelos innovadores, para adaptarlas a los futuros estudiantes (Barragán et al., 2012), que mejoran la relación estudiante-profesor, y motivan hacia el 
aprendizaje de las matemáticas. Aunado a esto, se puede mencionar la motivación del docente por usar metodologías activas (Lobato y Madinabeitia, 2011). Lo anterior justifica la necesidad de contar con programas con el apoyo teórico y práctico para los docentes en formación inicial (Costa et al., 2015).

Finalmente, la generalización de estos resultados es limitada debido al alcance de la intervención, especialmente al número bajo de participantes, así como otros factores contextuales (actividades de aprendizaje, características del estudiante y conocimiento del docente entre otros) que pueden entrar en juego, haciendo difícil, sino imposible, descartar todas las variables de confusión con respecto a cualquier resultado positivo que se encuentre. Por lo tanto, se hace necesario ampliar las intervenciones a más estudiantes y cursos ya que esto puede proporcionar evidencia adicional. Sin embargo, no deja de ser un estudio que suma a la creciente literatura del modelo aula invertida y alienta a los docentes e investigadores a experimentar cómo este modelo puede mejorar los cursos tradicionales.

\section{CONCLUSIONES}

De acuerdo con el trabajo presentado y a los resultados obtenidos, se pueden plantear las siguientes conclusiones:

1.- Este trabajo se suma a la creciente literatura sobre el aula invertida y alienta a los docentes e investigadores a experimentar cómo este modelo puede mejorar los cursos tradicionales, por un lado utilizando los principios FLIP, que actúan como una guía genérica para cambiar sus clases y mejorar el desempeño de los estudiantes y por otro, fundamentado en principios pedagógicos relacionados con el constructivismo fundamentado en la resolución de problemas, el aprendizaje activo y el aprendizaje colaborativo; el aprendizaje cooperativo y el aprendizaje experiencial asociado a los estilos de aprendizaje, para guiar el diseño de entornos de aprendizaje interactivos.

2.- Los resultados del estudio muestran una forma de invertir el aula en un curso de matemáticas a nivel universitario. Si bien se cree que la mejora en el rendimiento de los estudiantes fue el resultado del uso del aula invertida, se necesitan más evidencias para discernir si esta mejora se debió a su implementación.

3.- Los estudiantes reconocieron la conveniencia y los beneficios pedagógicos del enfoque aula invertida. Además, expresaron una alta preferencia por este enfoque en relación con el modelo tradicional dirigido por el docente. Esto les permitió reflexionar en dos perspectivas, por un lado, como estudiantes sobre el compromiso con su propio aprendizaje y la necesidad de desarrollarlo de manera grupal y autónoma y como futuros docentes, sobre la importancia de modernizar las prácticas pedagógicas con modelos innovadores.

4.- Los estudios futuros pueden proporcionar información adicional sobre el impacto del aula invertida en el rendimiento académico a medida que este modelo se adopta más en la práctica y la novedad disminuye.

5.- La generalización de los resultados es limitada debido al alcance de la intervención, especialmente al número relativamente bajo de participantes, así como otros factores contextuales que pueden entrar en juego, haciendo difícil, sino imposible, descartar todas las variables de confusión con respecto a cualquier resultado positivo que se encuentre. Por lo tanto, se hace necesario ampliar las intervenciones a más estudiantes y cursos ya que esto puede proporcionar evidencia adicional.

6.- A pesar de las limitaciones, este trabajo da un paso hacia la conceptualización del modelo aula invertida como un esfuerzo para transformar los cursos tradicionales a través de un proceso de IBD, y aun así ofrece perspectivas vitales sobre el potencial pedagógico de este modelo para la enseñanza de las matemáticas.

\section{AGRADECIMIENTOS}

Este artículo de investigación hace parte del proyecto "el aula invertida como estrategia pedagógica para desarrollar competencias matemáticas" financiado por la UFPS según contrato FINU 017-2018.

\section{REFERENCIAS}

Arévalo, M.A., García, M.A. y Hernández, C.A., Competencias TIC de los Docentes de Matemáticas en el Marco del Modelo TPACK, https://doi.org/10.22518/usergioa/jour/ccsh/2019.1/a07, Civilizar: Ciencias Sociales y Humanas, 19(36), 115-132 (2019).

Barab, S.A., Design-Based Research: A Methodological Toolkit for the Learning Scientis; in The Cambridge Handbook of the Learning Sciences by R. K. Sawyer (Ed.), Cambridge University Pres, pp. 153-169, New York, US (2006).

Bakker, A. y Van Eerde, D., An introduction to Design-Based Research with an example from Statistics Education, in Approaches to Qualitative Research in Mathematics Education, Springer, pp 429-466, New York (2015). 
Barragán, D.F., Gamboa, A.A. y Urbina, J.E., Práctica Pedagógica. Perspectivas Teóricas, Ecoe Ediciones, Bogotá (2010).

Bergmann, J. y Sams, A., Flip Your Classroom. Reach Every Student in Every Class Every Day, ISTE- ASCD, Washington, USA (2012).

Brown, A.L., Design experiments: Theoretical and Methodological Challenges in Creating Complex Interventions in Classroom Settings, https://doi.org/10.1207/s15327809jls0202_2, The Journal of the Learning Sciences, 2(2), $141-178$. (1992).

Cabı, E., The Impact of the Flipped Classroom Model on Students' Academic Achievement, https://doi.org/10.19173/irrodl.v19i3.3482, International Review of Research in Open and Distributed Learning, 19(3), 202221 (2018).

Carmona-Mesa, J.A. y Villa-Ochoa, J.A., Necesidades de Formación en Futuros Profesores para el Uso de Tecnologías. Resultados de un Estudio Documental, Paradigma, ISSN: 1011-2251, 38(1), 169-185 (2017).

Collins, A., Toward a Design Science of Education, in New directions in educational technology by E. Scanlon y T. O'Shea (Eds.), Springer-Verlag, pp 15-22, Berlin (1992).

Collins, B.V., Flipping the Precalculus Classroom, https://doi.org/10.1080/0020739X.2018.1535098, International Journal of Mathematical Education in Science and Technology, 50(5), 728-746 (2019).

Costa, L., Barros, V.F., Lopes, M.C., y Marques, L., La Formación Docente y la Educación de Jóvenes y Adultos: Análisis de la Práctica Pedagógica para la Enseñanza de Ciencias, https://dx.doi.org/10.4067/S0718-50062015000100002, Formación universitaria, 8(1), 03-12 (2015).

de Araujo, Z., Otten, S. y Birisci, S., Mathematics Teachers' Motivations for, Conceptions of, and Experiences with Flipped Instruction, https://doi.org/10.1016/j.tate.2016.11.006, Teaching and Teacher Education, 62, 60-70 (2017).

Design-Based Research Collective, Design-Based Research: An Emerging Paradigm for Educational Inquiry, https://doi.org/10.3102/0013189X032001005, Educational Researcher, 32(1), 5-8. (2003).

Durley, C., Flipped Classroom Transitions to Deep Learning, in Flipped learning: Gateway to student engagement by J. Bergmann y E. Sams, International Society for Technology in Education, pp. 1-182, Oregon, USA. (2014)

Gamboa, A.A., Calidad de la Educación Superior. Pretensiones y Realidades Institucionales, Ecoe Ediciones, Bogotá (2016).

Gaviria, D., Arango, J., Valencia, A. y Bran, L., Percepción de la Estrategia Aula Invertida en Escenarios Universitarios, Revista mexicana de investigación educativa, ISSN: 1405-6666, 24(81), 593-614 (2019).

Gravemeijer, K., Stephan, M., y otros tres autores, What Mathematics Education May Prepare Students for the Society of the Future? https://doi.org/10.1007/s10763-017-9814-6, International Journal of Science and Mathematics Education 15, 105-123 (2017).

Hamdan, N., McKnight, P., McKnight, K. y Arfstrom, K.M., The Flipped Learning Model: A White Paper Based on the Literature Review Titled. A Review of Flipped Learning, 1-16, Flipped LearningNetwork/Pearson/George Mason University (2013).

Hernández, C.A., Prada, R. y Ramírez, P., Competencias TIC e Investigativas entre Docentes de Educación Básica, Ecoe - Universidad Francisco de Paula Santander, Bogotá (2019).

Hung, H.T., Design-Based Research: Redesign of an English Language Course Using a Flipped Classroom Approach, https://doi.org/10.1002/tesq.328, Tesol Quarterly, 51(1), 180-192 (2017).

Kwan, C., Foon, K. y Chen, G., Toward a Set of Design Principles for Mathematics Flipped Classrooms: A Synthesis of Research in Mathematics Education, https://doi.org/10.1016/j.edurev.2017.08.002, Educational Research Review, 22, 5073 (2017).

Lobato, C. y Madinabeitia, A., Perfiles Motivacionales del Profesorado ante la Formación en Metodologías Activas en la Universidad, https://dx.doi.org/10.4067/S0718-50062011000100006, Formación universitaria, 4(1), 37-48 (2011).

Martín, D., Calvillo, A.J. y Tourón, J., The Flipped Learning: Guía "Gamificada" para Novatos y no tan Novatos, UNIR editorial (2017).

Molina, M., Castro, E., Molina, J.L. y Castro, E., Un acercamiento a la Investigación de Diseño a través de los Experimentos de Enseñanza, Enseñanza de las Ciencias, ISSN: 2174-6486, 29(1), 75-88 (2011).

Mendoza, S.M., Ramírez, P. y Gamboa, A.A., Argumentación en Escenarios Escolares. Aportes a la Formación de Maestros, Ecoe Ediciones, Bogotá (2019).

Mendoza, S.M., Hernández, C.A. y Prada, R., Principios de la Argumentación y las Prácticas Argumentativas en la Formación de Docentes en Matemáticas, Ecoe Ediciones - Universidad Francisco de Paula Santander, Bogotá (2019).

O'Flaherty, J. y Phillips, C., The Use of Flipped Classrooms in Higher Education: A Scoping Review, https://doi.org/10.1016/j.iheduc.2015.02.002, The Internet and Higher Education, 25, 85-95 (2015). 
Oliván B., Masluk, B., y otros cinco autores., The Use of Flipped Classroom as an Active Learning Approach Improves Academic Performance in Social Work: A Randomized trial in a University, https://doi.org/10.1371/journal.pone.0214623, PLoS One, 14(4) (2019).

Prada, R., Hernández, C.A. y Gamboa, A.A., Different scenarios for the teaching of mathematics with the support of virtual platforms: Flipped classroom, https://iopscience.iop.org/article/10.1088/1742-6596/1388/1/012046, Journal of Physics: Conference Series, 1388(1), 1-5 (2019a).

Prada, R., Hernández, C.A. y Gamboa, A.A., Usos y efectos de la implementación de una plataforma digital en el proceso de enseñanza de futuros docentes en matemáticas, https://doi.org/10.35575/rvucn.n57a10, Revista Virtual Universidad Católica del Norte, (57), 137-156 (2019b).

Prada, R., Hernández, C.A. y Ramírez, P., Enseñanza del Cálculo Diferencial: un Análisis de las Dificultades en Estudiantes Universitarios, Ecoe Ediciones - Universidad Francisco de Paula Santander, Bogotá (2020).

Rinaudo, M.C., y Donolo, D., Estudios de Diseño. Una Perspectiva prometedora en la Investigación Educativa, Revista de Educación a Distancia, ISSN: 1578-7680, (22), 1-29 (2010).

Rivero-Guerra, A.O., Práctica de Laboratorio de Granos de Almidón en un Curso de Botánica General: una Experiencia de Clase Invertida, http://dx.doi.org/10.4067/S0718-50062018000100087, Formación Universitaria, 11(1), 87-104 (2018).

Verkijika, S.F. y De Wet, L., Using a Brain-Computer Interface (BCl) in Reducing Math Anxiety: Evidence from South Africa, https://doi.org/10.1016/j.compedu.2014.10.002, Computers \& Education, 81, 113-122 (2015).

Turra, H., Carrasco, V., y otros tres autores, Flipped Classroom Experiences and their impact on Engineering Students' Attitudes Towards University-Level Mathematics, https://doi.org/10.1080/23752696.2019.1644963, Higher Education Pedagogies, 4(1), 136-155 (2019).

Zainuddin, Z. y Halili, S.H., Flipped Classroom Research and Trends from Different Fields of Study, https://doi.org/10.19173/irrodl.v17i3.2274, The International Review of Research in Open and Distributed Learning, 17(3), 313-340 (2016). 